\title{
Use of a CT statistical deformation model for multi-modal pelvic bone segmentation.
}

\author{
Steve Thompson ${ }^{1}$, Graeme Penney ${ }^{2}$, Damien Buie ${ }^{1}$, Prokar Dasgupta $^{3}$, Dave Hawkes ${ }^{1}$ \\ ${ }^{1}$ Centre for Medical Image Computing, University College London, United Kingdom \\ 2Interdisciplinary Medical Imaging Group, Kings College London \\ ${ }^{3}$ Department of Urology, Guy's and St Thomas' Hospital, London, United Kingdom
}

Copyright 2008 Society of Photo-Optical Instrumentation Engineers. This paper was published in SPIE Medical Imaging 2008 and is made available as an electronic reprint with permission of SPIE. One print or electronic copy may be made for personal use only. Systematic or multiple reproduction, distribution to multiple locations via electronic or other means, duplication of any material in this paper for a fee or for commercial purposes, or modification of the content of the paper are prohibited.

\begin{abstract}
We present a segmentation algorithm using a statistical deformation model constructed from CT data of adult male pelves coupled to MRI appearance data. The algorithm allows the semi-automatic segmentation of bone for a limited population of MRI data sets. Our application is pelvic bone delineation from pre-operative MRI for image guided pelvic surgery. Specifically, we are developing image guidance for prostatectomies using the daVinci telemanipulator. Hence the use of male pelves only. The algorithm takes advantage of the high contrast of bone in CT data, allowing a robust shape model to be constructed relatively easily. This shape model can then be applied to a population of MRI data sets using a single data set that contains both CT and MRI data. The model is constructed automatically using fluid based non-rigid registration between a set of CT training images, followed by principal component analysis. MRI appearance data is imported using CT and MRI data from the same patient. Registration optimisation is performed using differential evolution. Based on our limited validation to date, the algorithm may outperform segmentation using non-rigid registration between MRI images without the use of shape data. The mean surface registration error achieved was $1.74 \mathrm{~mm}$. The algorithm shows promise for use in segmentation of pelvic bone from MRI, though further refinement and validation is required. We envisage that the algorithm presented could be extended to allow the rapid creation of application specific models in various imaging modalities using a shape model based on $\mathrm{CT}$ data.
\end{abstract}

Keywords: segmentation, shape model, MRI, image guidance, registration, multi-modal

\section{INTRODUCTION}

Upcoming applications in image guided minimally invasive surgery will require the ability to rapidly segment and classify the large amount of information currently available from pre-operative patient scans. Currently the majority of segmentation is done manually by trained radiologists, coupling anatomical knowledge with the appearance of the scans to identify relevant physical structures. Due to the increasing amount of, and range of applications for, patient scan data there is a pressing need for fast, automatic segmentation methods.

The intended application for this segmentation is minimally invasive surgery on the prostate. We seek to construct a patient specific anatomical model prior to surgery. This model could have several applications including intraoperative guidance for the surgeon, surgical simulation prior to or during the procedure, or the determination of the optimum port placement of laparoscopic tools. ${ }^{1}$ The model can be registered to the patient in theatre using existing ultrasound based methods as utilised by Shao et al ${ }^{2}$ and Barratt et al. ${ }^{3}$ Our first target for segmentation is the pelvic bone. This large, rigid structure presents an excellent target for the intra-operative registration of the patient specific model, either through trans-rectal or freehand ultrasound. Knowledge of the

email: s.thompson@ucl.ac.uk web: http://www.cs.ucl.ac.uk/people/S.Thompson.html 
bone surface will also aid the subsequent segmentation of surrounding soft tissues using the bone to initialise anatomy models. ${ }^{4-6}$

Magnetic Resonance Imaging (MRI) data is gathered routinely for patients undergoing prostatectomies at Guy's Hospital, Computed Tomography (CT) data, however, is generally unavailable, nor can we justify an additional CT scan for the construction of an anatomical model. Therefore we are seeking to develop a method for the segmentation of the pelvic bone using MRI data only.

\subsection{Existing segmentation methods for bone in MRI.}

Several algorithms exist in the literature that aim to segment bone from MRI images, either in $2 \mathrm{D}$ or $3 \mathrm{D}^{4,7-9}$ without the use of prior shape knowledge. These approaches process the image intensities to create a measure of the local texture around each pixel/voxel. The consensus is that bone cannot be segmented using MRI intensity values directly due to overlapping intensities, intensity inhomogeneity over homogeneous anatomy due to scanning artefacts, and the lack of strong edges. Strzelecki ${ }^{7}$ and Carballido-Gamio et al ${ }^{8}$ use texture information to segment bony structure in the ankle and lower spine respectively. Kapur et al ${ }^{4}$ use region growing based on texture information followed by active contour smoothing to segment the femur and tibia. Lorigo et $a l^{9}$ use an active contour directly to segment the femur and tibia.

These algorithms will fail in conditions where the measure of image texture used is similar for bone and surrounding tissue. These failures are easily spotted using our understanding of what shape the bone should take. Building prior knowledge of the expected bone shape into the segmentation algorithm should therefore improve performance. Hoad and Martel $^{10}$ do this for the lower spine, modelling the spinal column as an elliptical column and masking data anterior to the spinal column. Cootes and Taylor ${ }^{11}$ mention the use of an active appearance model of the knee based on MRI data to segment bone. The shape of the pelvis has been successfully described using a statistical shape model by Lamecker et al, ${ }^{12}$ who used their model to accurately segment bone from CT images. Yao and Taylor ${ }^{13}$ incorporate CT intensity information into their pelvic bone model. Chan et $a l^{14}$ demonstrate the use of a pelvic CT statistical shape model for registration using ultrasound. We found no instances of the use of a pelvic bone shape model built or used with MRI data.

In this paper we combine shape information for the adult male pelvis derived from CT data with appearance information from an MRI volume. CT data has been used to construct the shape model due to the higher contrast of bone in CT data. This enables rapid, accurate, and largely automatic construction of the shape model without the need for segmentation of the bone in the training set. We use a patient data set containing both CT and MRI data for the pelvis to warp the MRI data to the mean shape of the model. This warped MRI appearance data can be used in conjunction with the modes of variation of the model to perform registrations with other MRI only data sets with similar appearance. Our shape model is built using similar methods to Heitz et $a l^{15}$ and Rueckert et al. ${ }^{16}$ We refer to the model as a statistical deformation model (SDM) as it is built using a set of deformation fields. Segmentation of the target MRI data sets can be achieved by performing a manual or semi-automatic segmentation of one CT data set in the SDM and warping this to each MRI set.

\section{METHOD}

Our method consists of four steps, these are:

- Build SDM using CT data.

- Warp MRI data to mean shape of SDM.

- Register MRI SDM to patient using the principal modes of variation.

- Measure registration errors and compare with alternative methods.

We first detail the data used, then describe each step in detail in the following sections. 


\subsection{Data.}

Anonymised data from 23 subjects was used in this study. $21 \mathrm{CT}$ only data sets were used to build the SDM. These covered the full pelvic bone except for the upper extreme of the iliac crests. This data had been previously acquired from male patients due to undergo hip replacement surgery. Areas within and adjacent to the femoral head showed variable amounts of disease/damage. These areas were not of interest to us and so were masked out. The areas of interest for our application (areas that may be used for intraoperative registration using ultrasound, specifically the inner face of the pubis or iliac crests) appeared to be unaffected, so we judged that the data, given the same sex and similar age profile, were relevant to our application. The data came from multiple scanners and was made up of scans of a variety of voxel sizes, see Table 1.

Two data sets consisting of CT and MRI data for the same patient were used, one to warp the MRI data to the model and the other for validation. These data sets came from patients undergoing brachytherapy for prostate cancer. These data sets covered a smaller region of the pelvis, (from the hip joints down). We would have preferred to validate our model on a more complete pelvis but do not have these data at present.

Table 1 gives a summary of the data used.

\begin{tabular}{|l|l|l|l|l|}
\hline Data & Type & Voxel Size (mm) & Volume & Used For \\
\hline $\begin{array}{l}\text { 21 Pelvis } \\
\text { Volumes }\end{array}$ & CT & $\begin{array}{l}\text { 0.7 to 0.9 In Plane (IP) } \\
\text { 2.0 to 3.2 Out of Plane (OP) }\end{array}$ & Full Pelvis & $\begin{array}{l}\text { SDM Construction } \\
\text { (Training Population) }\end{array}$ \\
\hline Patient A & CT \& MRI & $\begin{array}{l}\text { CT: 0.93 IP 3.0 OP } \\
\text { MRI: 0.7 IP 3.3 OP }\end{array}$ & $\begin{array}{l}\text { Lower Pelvis } \\
\text { (Below Hip Joint) }\end{array}$ & $\begin{array}{l}\text { Mapping MRI } \\
\text { Intensities to SDM }\end{array}$ \\
\hline Patient B & CT \& MRI & CT: 0.93 IP 3.0 OP & $\begin{array}{l}\text { Lower Pelvis } \\
\text { (Below Hip Joint) }\end{array}$ & Validation \\
& & MRI: 0.7 IP 3.9 OP & \\
\hline
\end{tabular}

Table 1. 23 Patient data sets were used in study. 21 CT data sets were used to build a SDM. 2 data sets containing CT and MRI data were used to apply the SDM to MRI data and validate its performance.

\subsection{CT statistical deformation model of male pelvis.}

Our SDM consists of the mean shape and the principal modes of population variation around the mean shape. To construct this it is necessary to know the correspondence between each data set in the training population. We found this by registering each data set in the training set to a single data set, which we will refer to as the reference data set (RDS). This was selected from the training population on the basis that its shape appeared to fall near the mean using visual inspection. During registration the mean shape of the model is used, so provided that the registrations to the RDS used to build the SDM are accurate the selection of the SDM should not bias the model. However this needs to be properly validated. We used a two stage registration, consisting of a 9 degree of freedom affine transform followed by a non rigid registration using deformations modelled as a compressible viscous fluid. Only these non rigid deformations were used to build the model. Figure 1 presents the model construction process as a flow chart.

\subsubsection{Affine registration to RDS.}

To focus the affine registration on bony areas of interest only, a bone mask was first created. This was done by manually segmenting the RDS. Neither the femurs nor the sacrum are of use for our intended application (registration to the inner face of the pubis or iliac crests) therefore these structures were excluded from the bone mask. Figure 2 shows the segmented bone shape for the entire pelvis.

The RDS was masked using a $4 \mathrm{~mm}$ dilation of this segmented bone, then the other 20 data sets were registered to the masked RDS with a 9 degree of freedom (DOF) affine transform (3 translations, 3 rotations and 3 scalings). The data sets were first manually aligned then each of the 9 DOFs varied iteratively to find the combination giving the optimum normalised mutual information (NMI). The accuracy of the registrations was checked by visual inspection. 


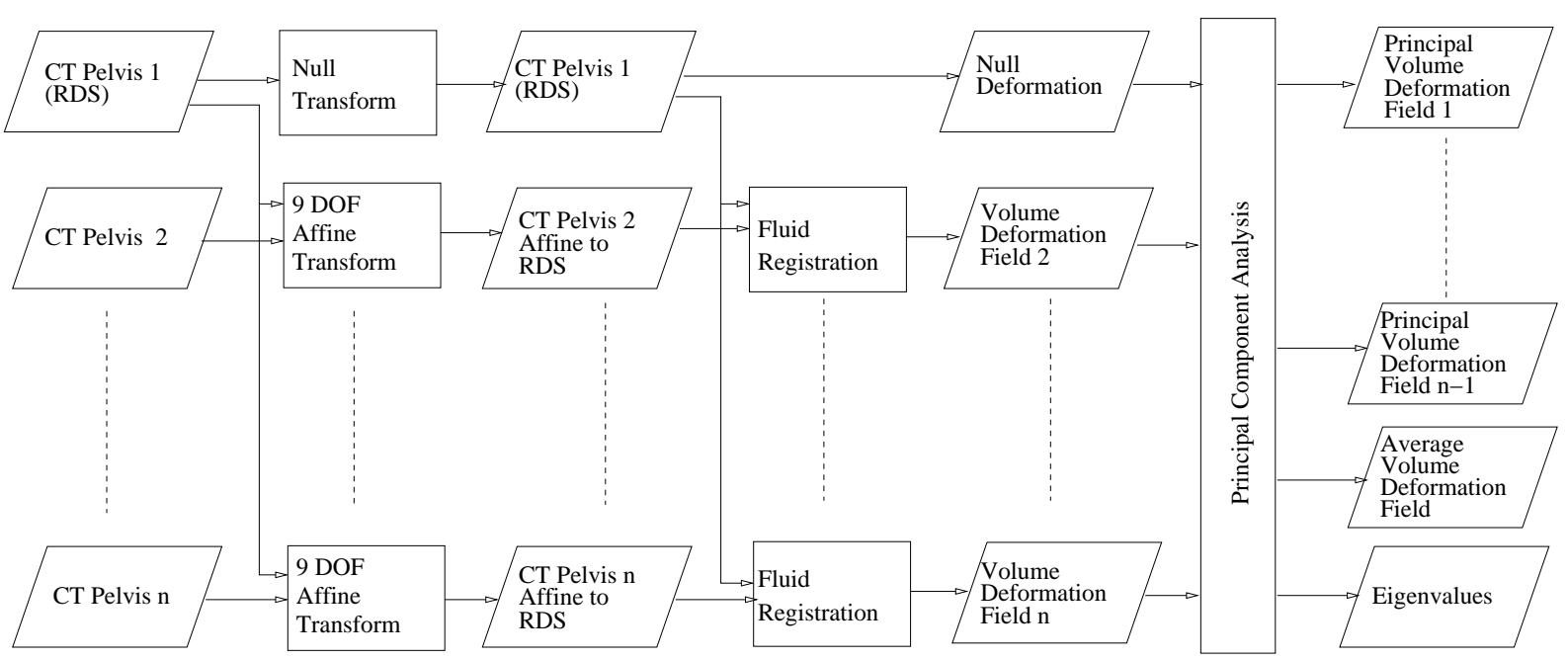

Figure 1. Flow chart showing the construction of the statistical deformation model. All data sets are first brought into alignment using a 9 DOF (3 translations, 3 rotations, 3 scalings) registration. Non rigid registration based on compressible viscous fluid flow is then used to determine the correspondence between each data set. We chose to drive both the affine and non rigid registrations using normalised mutual information. Principal component analysis is used on these deformation fields to give a set of principal deformation fields and a mean shape. Processing steps are shown as rectangles, inputs/outputs as parallelograms.

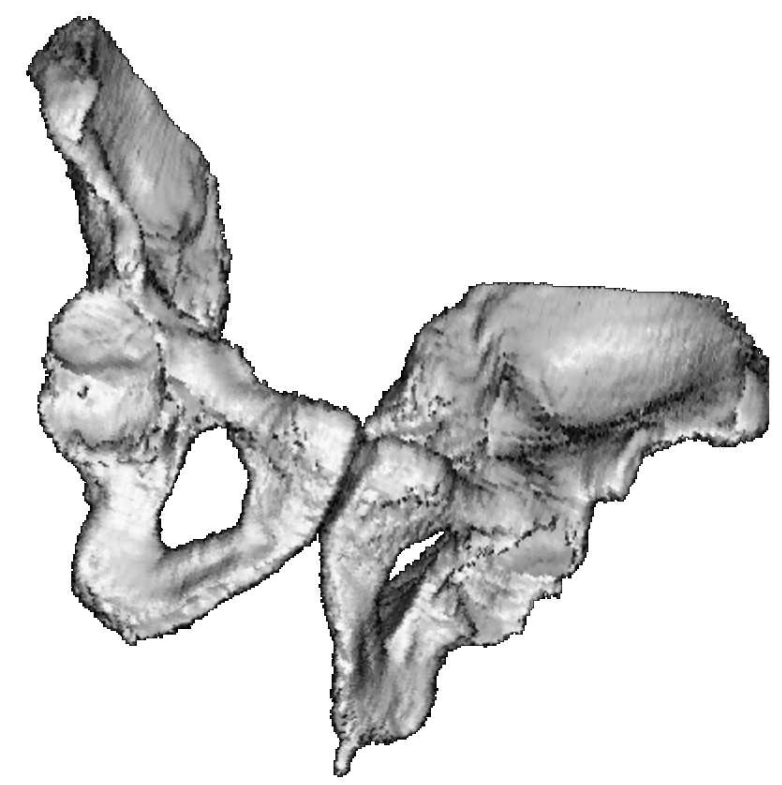

Figure 2. The segmented full pelvis showing which areas of bone were used in the construction of the SDM. This was dilated by $4 \mathrm{~mm}$ to provide a mask containing the bone of interest and the immediate surrounding tissue.

\subsubsection{Non rigid registration using compressible viscous fluid flow.}

The resulting 20 volumes were then non rigidly registered to the RDS using a fluid registration algorithm developed by Crum et al. ${ }^{17}$ This models a displacement field on the target image (the RDS) as a compressible viscous fluid. In this case NMI between this and the source image is used to drive the fluid flow to optimise NMI. This provides a transformation without folding or tearing, ie. diffeomorphic. A 3 stage multi-resolution approach was used, quarter, half, then full resolution. A $30 \mathrm{~mm}$ dilation around the segmented RDS bone was 


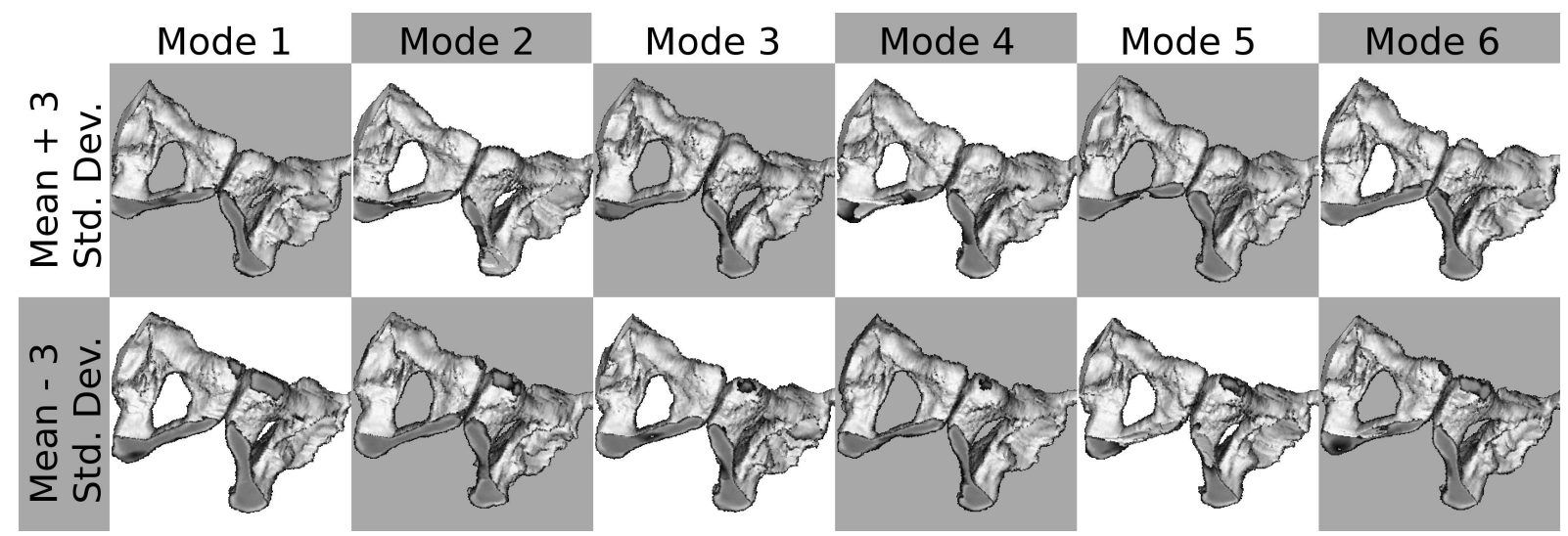

Figure 3. First 6 principal components of training data set. These are ranked based on their contribution to the population variance. Note the deformation fields used to make these have been cropped to below the hip joint.

used as a mask to prevent tissue distant from any bone surfaces being included in the registration. This mask size ensured that all relevant bone in the data set was captured. The quality of the registrations was checked by visual inspection. The output from each registration is a 3D deformation field at the same resolution and size as the target image showing the deformations required to transform the source to the target. In our case therefore we are left with 20 deformation fields, all at the same resolution and size, that define the non rigid correspondences between the data in our training set. These deformation fields form the basis of the SDM.

\subsubsection{Principal component analysis of deformation fields.}

Principal component analysis was performed on the 20 deformation fields plus the null deformation for the RDS. The output of this was an average deformation field that defines the mean shape of the population (deformed from the RDS) along with 20 deformation fields defining 20 orthogonal modes of variation about this mean. These were ranked in order of the amount of population variance they account for. The first 6 of these modes are shown in figure 3. It should be noted that as mentioned in Section 2.1, the data sets used to validate this method are smaller than the full pelvic volumes used to create the deformation fields in the previous section. For this reason the deformation fields were first cropped to a volume closer to the validation data sets. Hence the incomplete pelves seen in figure 3. It was decided that doing this enabled a more compact model while still enabling the construction of a full pelvic SDM when suitable validation data is acquired.

Figure 4 shows the cumulative variance plot for all 20 principal components. The first mode accounts for $17 \%$ of the training set variance. As will be discussed in Section 2.4 we elected to use the first 12 modes of variation in our registration algorithm. These account for $86 \%$ of the training set variation.

\subsection{Warping MRI appearance data to SDM.}

Figure 5 shows how MRI appearance data was built into our CT based SDM. The method requires matched $\mathrm{CT}$ and MRI for the region of interest. We used a 9 DOF affine registration to align the CT and MRI data. The three directional scaling should account for calibration errors on either piece of equipment. In this case the quality of the registration was evaluated by comparing manual segmentations of both the CT and the MRI data. The CT volume was registered to the RDS using the technique of an affine registration followed by a fluid registration as for the construction of the SDM, see Section 2.2. The resulting affine transformation matrix and deformation field were applied to the matched MRI data to transform the MRI volume to the RDS. The next section details how this warped MRI volume, the deformation to the mean shape, and the 20 modes of variation about this can be used to perform a registration.

\subsection{Validation of method.}

To assess the performance of the proposed method 4 registrations were performed, these are detailed in figure 6 . An explanation of each registration follows. 


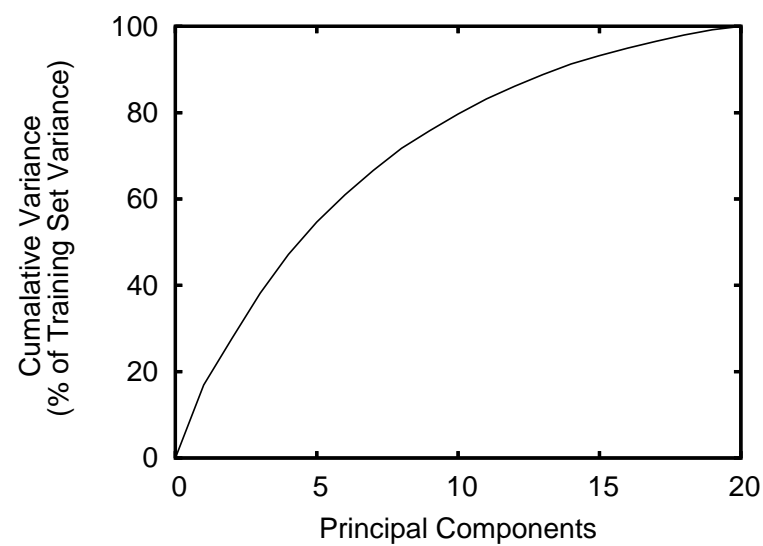

Figure 4. Cumulative percentage of population variance accounted for by the 20 principal components. The first mode accounts for $17 \%$ of the training set variance and the first 12 modes account for $86 \%$ of the training set variance.

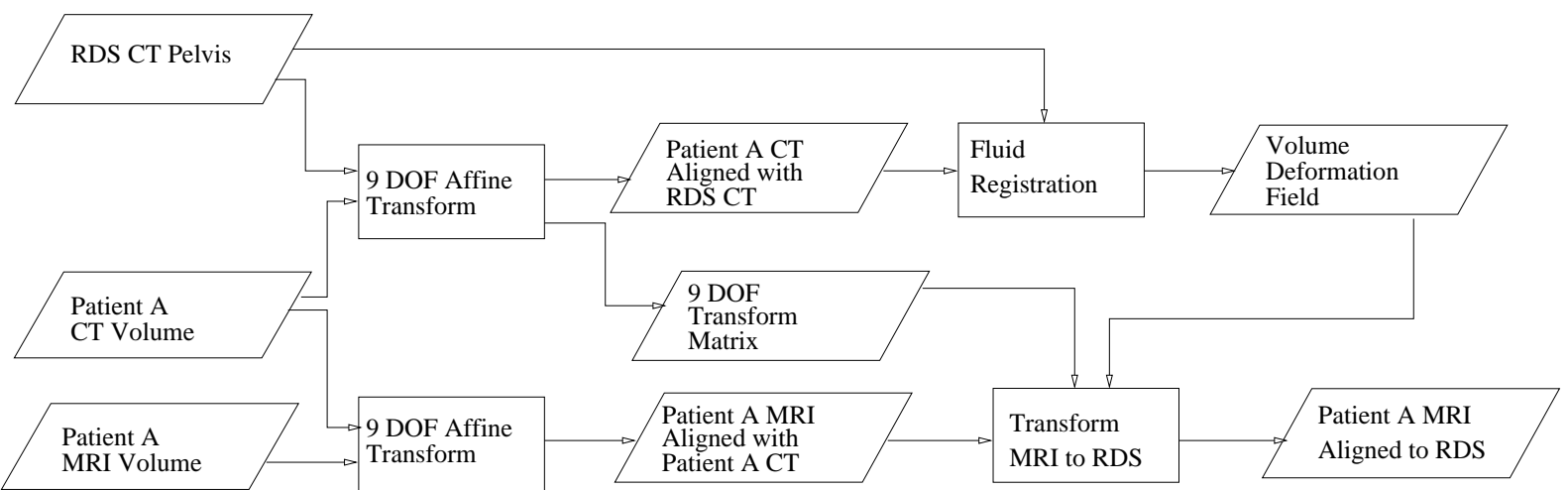

Figure 5. Flow chart showing method used to warp MRI data to reference data set used in SDM. This can be easily warped to the SDM mean shape by applying the SDM average deformation. Patient CT data is non rigidly aligned to the RDS CT data and the resulting deformation applied to matching patient MRI data. Registration between the MRI and CT data uses a 9 DOF affine registration. This may account for calibration errors in either data set. Processing steps are shown as rectangles, inputs and outputs as parallelograms.

\subsubsection{Registration 1 (R1): CT to CT using SDM.}

This registration is intended to evaluate the performance of the SDM independently of any effects due to the warping and use of the MRI data. The target image is defined as the source image deformed by the SDM's average deformation plus a linear combination of the first 12 deformation modes. In theory any of the 20 modes of variation can be used here. We selected the first 12 as they accounted for most of the variation in the training set. Selecting less will result in a faster algorithm, while selecting more will give a more complete description of the geometry of the training set. Determination of the optimum set of modes to use would require more validation data sets than we had available.

We elected to continue using NMI as the similarity measure between the deformed source and the target. The NMI calculation was restricted via a region mask of $2 \mathrm{~mm}$ around the segmented bone mask, see Section 2.2.1, to prevent regions away from the bone influencing the registration. This similarity measure is used to drive a differential evolution ${ }^{18}$ algorithm to generate a set of coefficients for the 12 modes that optimises the NMI.

We set starting estimates for the 12 coefficients to be within +/- 3 standard deviations of the population mean for each mode. The coefficients used for each member of the starting population were set within these limits using a pseudo random number generator. However there was no hard constraint on the coefficients to prevent them evolving to values outside this range, i.e. to values that would not be predicted by the training 


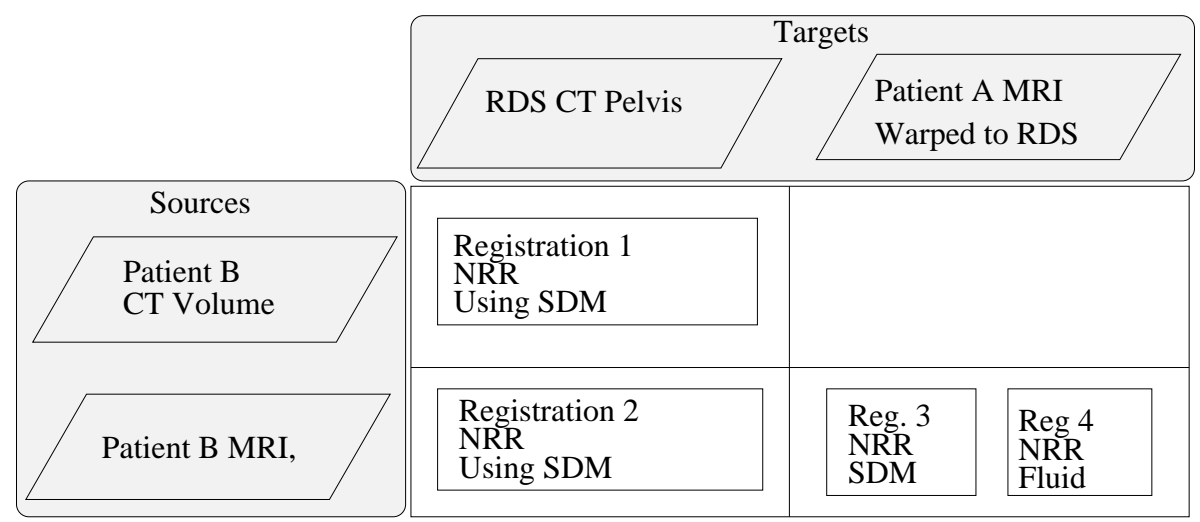

Figure 6. Registrations used in validation process. Both sources were first aligned to the RDS with a 9 DOF affine transform. The same affine transform was used for all registrations using the MRI source. A Gaussian blur was applied to the MRI source prior to registration. (NRR - Non Rigid Registration)

set. We feel this is appropriate as given the small size of the training set it is unlikely to be representative of the actual patient population. Validation on a larger sample would be required to justify this assumption or otherwise.

\subsubsection{Registration 2 (R2): MRI to CT using SDM.}

This registration is intended to show whether warping the MRI data to the SDM provides a measurable benefit. That is, as NMI is a multi-modal similarity measure, could the same results be achieved by registering the MRI data direct to the CT RDS. The method used was the same as for Registration 1 excepting the following 3 changes.

- Patient B MRI data set was used as the source image. A Gaussian blur with a kernel size of $0.75 \mathrm{~mm}$ was first applied to this image.

- The mask used to calculate the NMI was expanded to the segmented bone plus $10 \mathrm{~mm}$.

- The affine transform used for initial alignment was calculated from the two MRI images, whereas the 2 CT images were used for Registration 1.

\subsubsection{Registration 3 (R3): MRI to MRI using SDM.}

This is our proposed optimal method, using the SDM and the warped MRI appearance data. The method used is identical to Registration 2 except the warped Patient A MRI data was used as the target image.

\subsubsection{Registration 4 (R4): MRI to MRI using fluid registration.}

Here we are attempting to see if the SDM algorithm provides any benefit over a non rigid registration using the same fluid registration method described in Section 2.2.2. The source and target are as for Registration 3.

\subsubsection{Registration evaluation.}

The performance of each registration was assessed by inverting the deformation field generated and using this to deform the manual segmentation of the RDS to the source image. This gave a binary bone segmentation that could be compared to a manual segmentation of either the Patient B CT data (Registration 1) or the Patient B MRI data (Registrations 2-4), referred to here as the gold standards. Two measures of the registration accuracy were used;

- the average distance from each point on the registered segmented surface to the nearest point on the gold standard segmentation surface, and 
- the percentage of the registered segmented volume that is overlapped by the gold standard volume.

In each case we also compared these values to those of the case where there was no non rigid registration, ie. the data sets were aligned by the 9 DOF affine transform only.

\section{RESULTS}

Table 2 shows quantitative results for each of the 4 registration methods. Figure 7 shows the segmentation results for the MRI SDM based method (Registration 3) on three example axial slices through Patient B MRI data.

\begin{tabular}{|l|l|l|l|l|}
\hline Reg. Name & R1 & R2 & R3 & R4 \\
\hline Average Distance between Surfaces (mm) & 1.68 & 1.95 & 1.74 & 1.86 \\
Average Distance for Affine only Registration (mm) & 1.81 & 1.75 & 1.75 & 1.75 \\
\hline Overlap of Segmented Bone with Gold Standard (\%) & 87.16 & 85.80 & 85.70 & 80.22 \\
Overlap Using Affine only Registration (\%) & 83.82 & 81.04 & 81.04 & 81.04 \\
\hline
\end{tabular}

Table 2. Average surface distance and percentage volume overlaps between automatic and manual (gold standard) bone surface segmentations for 4 registration methods used. Changes from measures calculated using only affine registration are shown in the second row of each section.

\section{DISCUSSION}

Table 2 shows that for the registrations to MRI data our proposed method (R3) was the most accurate. However the differences were marginal, and because we currently only have a single data set for validation there is little basis for making any claims on whether one method is superior to any of the others based on this data set. The fact that none of the methods show a significant improvement over the affine only transform suggests that the data set we used for validation was very close to the RDS and thus did not not adequately demonstrate the non rigid registrations. More complete validation is in progress pending more data. We believe that the shape information present in the SDM should aid the registration and this will be demonstrable when larger non rigid deformations are required. Based on the results we presented here we have demonstrated the feasibility of our approach, but it is not realistic to draw any further conclusions. We confirmed that the differential evolution algorithm was evolving to a solution with an improved NMI to the starting estimate. For the MRI model solution, the absolute variation of the SDM components was on average 0.98 standard deviations away from the mean, with the largest mode deformation being 2.07 standard deviations. The results for registrations 1 and 3 are similar indicating that our method of incorporating MRI appearance information into the SDM is valid in this case.

The SDM contains shape data from a population of 21 CT scans and MRI appearance data from 1 subject. The SDM should be applicable to patient data that falls near the shape range covered by the CT model training data and is acquired with the same MRI protocol. Work by van de Kraats et al ${ }^{19}$ indicates that MRI intensities are similar across scans for a small region of interest. Based on this our model should work for our application (patients due to undergo robot-assisted radical prostatectomy at a single institution). Further validation is required to confirm this. The method needs to be validated with a larger population, using data covering a more complete pelvic volume, this work is in progress. It may then become necessary to incorporate MRI appearance information from multiple subjects, or to transform the MRI intensities to a localised texture measure.

The segmentation examples shown in Figure 7 show a plausible segmentation. The method presented here allows for the creation of application specific SDMs from a generic CT derived SDM. The application specific SDM can be created rapidly using data in both modalities from the same subject. The method is generic and could in principle be applied to any situation in which a model is built from one modality (i.e. the modality that shows the required structure with optimal contrast), but instantiated with another modality (i.e. the only one available). 


\section{ACKNOWLEDGMENTS}

We are grateful for the support of EPSRC grants GR/EP/D033969 and GR/T02799/03 together with DTI technology program grant 1612 "macnavos".

We also acknowledge DePuy Orthopaedics Inc. who supplied the data for the shape model.

\section{REFERENCES}

1. L. Adhami and E. Coste-ManiEre, "Optimal planning for minimally invasive surgical robots," IEEE Transactions on Robotics and Automation 19, pp. 854-863, Oct. 2003.

2. W. Shao, R. Wu, K. V. Ling, C. H. Thng, H. S. S. Ho, C. W. S. Cheng, and W. S. Ng, "Evaluation on similarity measures of a surface-to-image registration technique for ultrasound images," in Medical Image Computing and Computer Assisted Intervention, R. Larsen, M. Nielsen, and J. Sporring, eds., LNCS 4191, pp. 742-749, Springer, 2006.

3. D. Barratt, G. Penney, C. Chan, M. Slomczykowski, T. Carter, P. Edwards, and D. Hawkes, "Self-calibrating 3D-ultrasound-based bone registration for minimally invasive orthopedic surgery," IEEE Transactions on Medical Imaging 25, pp. 312-323, March 2006.

4. T. Kapur, P. Beardsley, S. Gibson, W. Grimson, and W. Wells, "Model-based segmentation of clinical knee MRI," in IEEE Int'l Workshop on Model-Based 3D Image Analysis, pp. 97-106, 1998.

5. A. Tsai, W. Wells, C. Tempany, E. Grimson, and A. Willsky, "Mutual information in coupled multi-shape model for medical image segmentation.," Medical Image Analysis 8, pp. 429-445, Dec 2004.

6. D. Shen and C. Davatzikos, "Adaptive-focus statistical shape model for segmentation of 3D MR structures," in Medical Image Computing and Computer Assisted Intervention, S. L. Delp, A. M. DiGioia, and B. Jaramaz, eds., LNCS 1935, pp. 206-215, Springer, 2000.

7. M. Strzelecki, "Texture boundary detection using network of synchronised oscillators," Electronics Letters $\mathbf{4 0}$ (8), pp. 466-467, 2004.

8. J. Carballido-Gamio, S. Belongie, and S. Majumdar, "Normalized cuts in 3-D for spinal MRI segmentation," IEEE Transactions on Medical Imaging 23, pp. 36-44, Jan. 2004.

9. L. M. Lorigo, O. Faugeras, W. E. L. Grimson, R. Keriven, and R. Kikinis, "Segmentation of bone in clinical knee MRI using texture-based geodesic active contours," in Medical Image Computing and Computer Assisted Intervention, W. M. Wells, A. C. F. Colchester, and S. L. Delp, eds., LNCS 1496, pp. 1195-1204, Springer, 1998.

10. C. L. Hoad and A. L. Martel, "Segmentation of MR images for computer-assisted surgery of the lumbar spine," Physics in Medicine \&3 Biology 47, no 19, pp. 3503-3517, 2002.

11. T. F. Cootes and C. J. Taylor, "Anatomical statistical models and their role in feature extraction," British Journal of Radiology 77(2), pp. S133-S139(1), 2004.

12. H. Lamecker, M. Seebass, H. C. Hege, and P. Deuflhard, "A 3D statistical shape model of the pelvic bone for segmentation," in Proceedings of the SPIE Medical Imaging 2004: Image Processing., J. M. Fitzpatrick and M. Sonka, eds., 5370, pp. 1341-1351, May 2004.

13. J. Yao and R. H. Taylor, "Non-rigid registration and correspondence finding in medical image analysis using multiple-layer flexible mesh template matching.," International Journal of Pattern Recognition and Artificial Intelligence 17(7), pp. 1145-1165, 2003.

14. C. Chan, D. Barratt, P. Edwards, G. Penney, M. Slomczykowski, T. Carter, and D. Hawkes, "Cadaver validation of the use of ultrasound for $3 \mathrm{D}$ model instantiation of bony anatomy in image guided orthopaedic surgery," in Medical Image Computing and Computer Assisted Intervention, 3217, pp. 397-404, 2004.

15. G. Heitz, T. Rohlfing, and C. R. Maurer, Jr., "Statistical shape model generation using nonrigid deformation of a template mesh," in Proceedings of the SPIE Medical Imaging 2005: Image Processing, J. M. Fitzpatrick and J. M. Reinhardt, eds., 5747, pp. 1411-1421, Apr. 2005.

16. D. Rueckert, L. I. Sonoda, C. Hayes, D. L. G. Hill, and M. O. Leach, "Nonrigid registration using free-form deformations: Application to breast MR images," IEEE Transactions on Medical Imaging 18, pp. 712-721, Aug. 1999. 
17. W. R. Crum, C. Tanner, and D. J. Hawkes, "Anisotropic multi-scale fluid registration: evaluation in magnetic resonance breast imaging," Physics in Medicine and Biology 50, pp. 5153-5174, 2005.

18. K. Price, R. Storn, and J. Lampinen, Differential Evolution - A Practical Approach to Global Optimization, Springer, ISBN: 3-540-20950-6, 2005.

19. E. B. van de Kraats, G. P. Penney, T. van Walsum, and W. J. Niessen, "Multispectral MR to X-ray registration of vertebral bodies by generating CT-like data.," in Medical Image Computing and Computer Assisted Intervention, J. S. Duncan and G. Gerig, eds., LNCS 3750, pp. 911-918, Springer, 2005. 

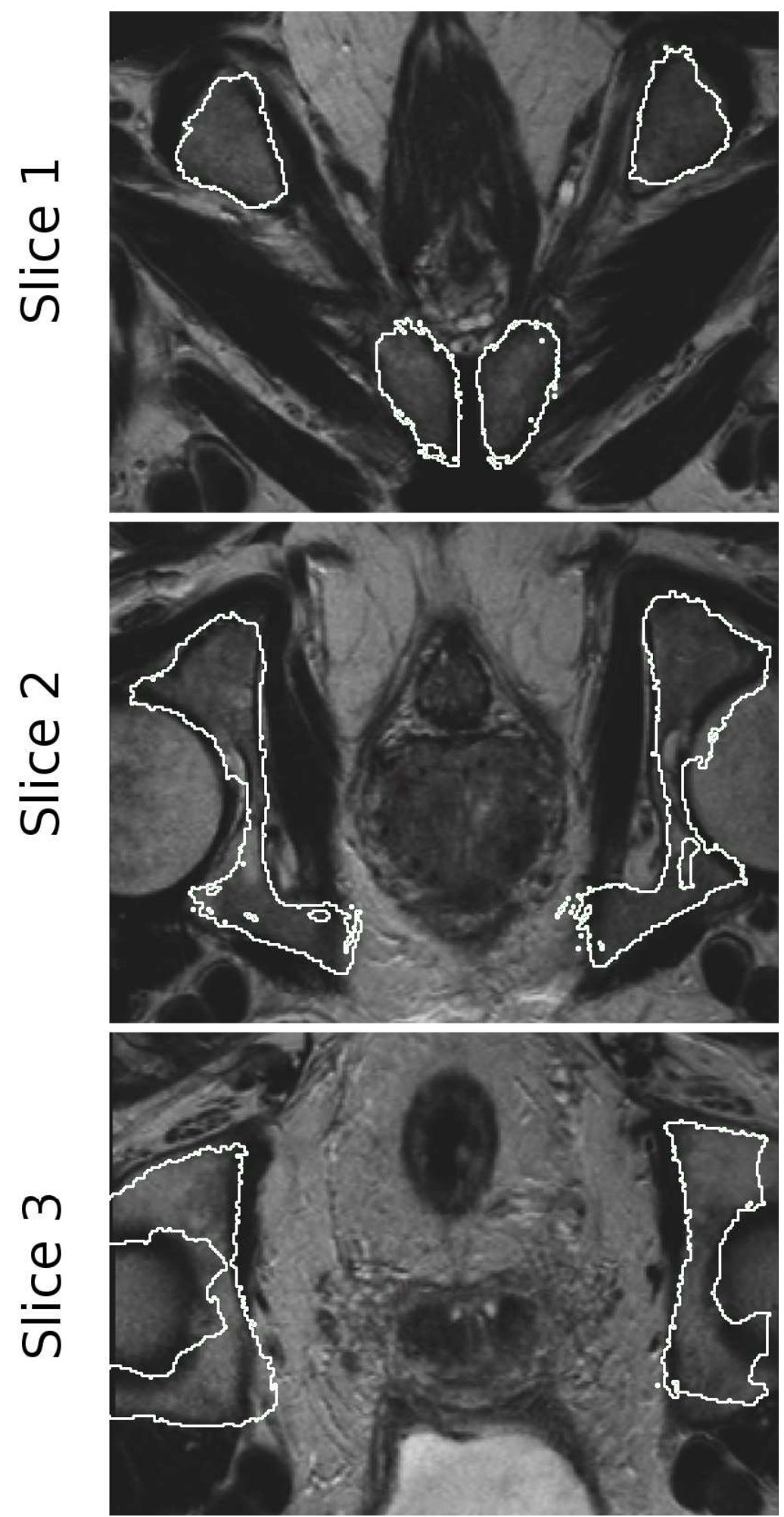

Figure 7. Results of registration algorithm (Registration 3) shown as bone segmentation boundary made by applying inverse deformation to manual segmentation of RDS. Results shown for 3 example axial slices. 